\title{
Impact of the mesoscale range on error growth and the limits to atmospheric predictability
}

Article

Accepted Version

Leung, T. Y. ORCID: https://orcid.org/0000-0003-0056-284X, Leutbecher, M., Reich, S. and Shepherd, T. G. (2020) Impact of the mesoscale range on error growth and the limits to atmospheric predictability. Journal of the Atmospheric Sciences, 77 (11). pp. 3769-3779. ISSN 1520-0469 doi: https://doi.org/10.1175/JAS-D-19-0346.1 Available at https://centaur.reading.ac.uk/92340/

It is advisable to refer to the publisher's version if you intend to cite from the work. See Guidance on citing.

To link to this article DOI: http://dx.doi.org/10.1175/JAS-D-19-0346.1

Publisher: American Meteorological Society

All outputs in CentAUR are protected by Intellectual Property Rights law, including copyright law. Copyright and IPR is retained by the creators or other copyright holders. Terms and conditions for use of this material are defined in the End User Agreement. 


\section{CentAUR}

Central Archive at the University of Reading

Reading's research outputs online 


\title{
Impact of the mesoscale range on error growth and the limits to
}

\section{atmospheric predictability}

\author{
Tsz Yan Leung* \\ Department of Mathematics and Statistics, University of Reading, United Kingdom \\ Martin Leutbecher \\ European Centre for Medium-range Weather Forecasts, Reading, United Kingdom \\ Sebastian Reich \\ Institute of Mathematics, University of Potsdam, Germany \\ Department of Mathematics and Statistics, University of Reading, United Kingdom
}

Department of Meteorology, University of Reading, United Kingdom

${ }_{12}^{*}$ Corresponding author address: Department of Mathematics and Statistics, Whiteknights, PO Box

13 220, Reading RG6 6AX, United Kingdom

${ }_{14}$ E-mail: tsz.leung@ @gr.reading.ac.uk 


\section{ABSTRACT}

Global numerical weather prediction (NWP) models have begun to resolve ${ }_{16}$ the mesoscale $k^{-\frac{5}{3}}$ range of the energy spectrum, which is known to impose an ${ }_{17}$ inherently finite range of deterministic predictability per se as errors develop ${ }_{18}$ more rapidly on these scales than on the larger scales. However, the dynam${ }_{19}$ ics of these errors under the influence of the synoptic-scale $k^{-3}$ range is little studied. Within a perfect-model context, the present work examines the error ${ }_{21}$ growth behavior under such a hybrid spectrum in Lorenz's original model of 1969, and in a series of identical-twin perturbation experiments using an idealized two-dimensional barotropic turbulence model at a range of resolutions.

${ }_{24}$ With the typical resolution of today's global NWP ensembles, error growth 25 remains largely uniform across scales. The theoretically expected fast error 26 growth characteristic of a $k^{-\frac{5}{3}}$ spectrum is seen to be largely suppressed in the ${ }_{27}$ first decade of the mesoscale range by the synoptic-scale $k^{-3}$ range. However, ${ }_{28}$ it emerges once models become fully able to resolve features on something ${ }_{29}$ like a 20-kilometer scale, which corresponds to a grid resolution on the order 30 of a few kilometers. 


\section{Introduction}

The idea that the Earth's atmosphere possesses an inherently finite limit to deterministic predictability has been a universally accepted fact in dynamical meteorology since Lorenz (1969) demonstrated it using a simple turbulence model. He argued that the predictability of a flow depends on the slope of the energy spectrum $E(k)$ (the spectral slope), where $k$ is the scalar wavenumber: flows with spectra shallower than $k^{-3}$ have limited predictability as the scale of the initial error decreases, whereas those with spectra steeper than $k^{-3}$ are indefinitely predictable (assuming a perfect model) as long as the initial error is small enough in scale. Arguing that the atmospheric spectrum behaves as $k^{-\frac{5}{3}}$, he concluded that atmospheric predictability is inherently limited.

It was subsequently realized that the large-scale atmospheric flow follows a $k^{-3}$ energy spectrum (Boer and Shepherd 1983), consistent with the expectations of two-dimensional (2D) turbulence forced at the large scales. With the aid of aircraft observations, Nastrom and Gage (1985) showed that the $k^{-3}$ range transitions into a $k^{-\frac{5}{3}}$ range in the mesoscale, at a wavelength of about 400 kilometers. This does not change Lorenz's conclusion of limited predictability, as the latter depends on the spectral slope in the high-wavenumber limit. Recent studies with realistic numerical weather prediction (NWP) models continue to find that deterministic predictability is limited to about 2 to 3 weeks, as Lorenz suggested (Buizza and Leutbecher 2015; Judt 2018).

In recent years, thanks to ever-increasing computational power, atmospheric models have started to resolve the $k^{-\frac{5}{3}}$ range, where the flow becomes increasing three-dimensional. Moist processes such as convection and clouds that are thought to impose an intrinsic barrier to predictability (Sun and Zhang 2016) are now partially or explicitly resolved. However, the interplay between the synoptic-scale $k^{-3}$ and mesoscale $k^{-\frac{5}{3}}$ ranges has been little studied. In particular, it was not 
so clear whether the error growth would resemble characteristics of the $k^{-3}$ or $k^{-\frac{5}{3}}$ paradigm, until Judt (2018) reported, using a full global NWP model, that error growth was fairly uniform across scales - a feature of $k^{-3}$ turbulence. Judt's study suggests that error growth and hence predictability properties under the hybrid spectrum are not as straightforward as might be thought. It also provokes questions on the sensitivity of such properties to the resolution of the model. Therefore, it is essential to assess the impact of the synoptic-scale $k^{-3}$ range on error growth in the mesoscale $k^{-\frac{5}{3}}$ range and to understand its sensitivity to the extent to which the mesoscale range is resolved.

Such a study must be done at the expense of the complexity of model dynamics, as limited computational resources make it infeasible to be done with a full NWP model. The much simpler 2D barotropic vorticity model has been used in a number of previous turbulence and predictability studies (Maltrud and Vallis 1991; Rotunno and Snyder 2008; Durran and Gingrich 2014), among which Rotunno and Snyder (2008) demonstrated that the model dynamics per se has limited impact on the predictability properties of a turbulent flow; instead, the error growth and predictability properties are largely determined by the shape of the energy spectrum. In light of this, it is justified to perform predictability experiments under the hybrid $k^{-3}$ and $k^{-\frac{5}{3}}$ spectrum with the barotropic model and Lorenz's original error growth model of 1969 (also based on the barotropic model), which can be run at higher resolutions and thereby resolve a substantially more extensive part of the mesoscale $k^{-\frac{5}{3}}$ range. The choice of these simple models is in no way intended to downplay the role of the three-dimensional mesoscale processes in limiting predictability; these effects are, rather, collectively included in the $k^{-\frac{5}{3}}$ range. The use of these models is simply motivated by their ability to facilitate predictability experiments at unprecedentedly high resolutions so as to gain insights into the error growth and predictability properties associated to these fine scales. 
This article investigates the behavior of error growth under the canonical hybrid $k^{-3}-k^{-\frac{5}{3}}$ spectrum, and demonstrates that the synoptic-scale $k^{-3}$ range exerts an influence on the first decade of the mesoscale range by largely suppressing the fast upscale cascade of error energy characteristic of a $k^{-\frac{5}{3}}$ spectrum. It is structured as follows. Section 2 presents a systematic set of identical-twin perturbation experiments with the $2 \mathrm{D}$ barotropic vorticity model at a range of resolutions. Section 3 introduces a scale-dependent parametric error growth model, one of whose parameters provides information on the error growth rate, so that its dependence on the physical length scale can be analyzed. Section 4 demonstrates that the error growth behavior in the $2 \mathrm{D}$ barotropic vorticity model can be captured by the even simpler model of Lorenz (1969), which is then used to assess how the results would change in the infinite-resolution limit. Section 5 examines the sensitivity of the results to the initial error profile. Finally, Section 6 summarizes and concludes the paper.

\section{Identical-twin perturbation experiments with a 2D barotropic vorticity model}

\section{a. The model and experimental design}

Two sets of perturbation experiments are performed on a forced-dissipative version of the dimensionless 2D barotropic vorticity model

$$
\frac{\partial \theta}{\partial t}+J(\psi, \theta)=f+d, \quad \theta=\Delta \psi
$$

in a doubly periodic domain, where $\psi$ is the velocity streamfunction [related to the velocity $\boldsymbol{u}$ by $\boldsymbol{u}=-\nabla \times(\psi \hat{\mathbf{k}})], \Delta=\nabla \cdot \nabla, \nabla=\left(\frac{\partial}{\partial x}, \frac{\partial}{\partial y}\right)$ and $J(A, B)=\frac{\partial A}{\partial x} \frac{\partial B}{\partial y}-\frac{\partial A}{\partial y} \frac{\partial B}{\partial x}$. The prognostic variable of the model is the vorticity $\theta$. The model is run pseudo-spectrally at various resolutions $k_{t} \in$ $\{256,512,1024,2048\}$ (where $k_{t}$ is the truncation wavenumber), and the forcing $f$ and dissipation $d$ are prescribed in spectral space. 
Before the perturbations are applied, the turbulence is spun up to a statistically stationary state so that the energy spectra have the desired shapes which do not significantly change in time. To generate a $k^{-3}$ spectrum transitioning into $k^{-\frac{5}{3}}$ at a smaller scale, forcing is applied at both large and small scales. This allows both a direct enstrophy cascade and an inverse energy cascade. Following Maltrud and Vallis (1991), the simulations are forced at wavevectors whose modulus $k$ falls within the ranges $[10,14]$ and $\left[\frac{5}{8} k_{t}, \frac{165}{256} k_{t}\right]$. The former represents synoptic-scale baroclinic forcing, and the latter mesoscale forcing, which is applied at a small undamped scale and hence depends on $k_{t}$. Independently for each $2 \mathrm{D}$ wavevector in these wavebands, $f$ is controlled by the complex-valued stochastic process

$$
\mathrm{d} f=-\frac{1}{t_{f}} f \mathrm{~d} t+\hat{A} \sqrt{\frac{2}{t_{f}}} \mathrm{~d} \tilde{W}
$$

which is an Ornstein-Uhlenbeck process except that the noise $\tilde{W}$ is a uniform random number on the unit circle in the complex plane. The $e$-folding de-correlation time $t_{f}$ is fixed at 0.5 across experiments of different resolutions, whereas the standard deviation of the forcing amplitude $\hat{A}$ depends on the forced waveband and the resolution (more on this later).

Dissipation is introduced to remove the energy and enstrophy cascaded into the largest and smallest scales respectively. At the largest scales $k \in[1,3]$, the dissipation comes in the form of a linear drag $d=-0.0029 \theta$. At the smallest scales $k \geq \frac{25}{32} k_{t}, d=-0.083 \Delta^{8} \theta$, which is a hyperviscosity. It is worth emphasizing that for most wavenumbers both the forcing and dissipation are absent. This enables clean energy and enstrophy cascades along the inertial ranges.

To mimic real-world models which do not compromise the quality of large-scale predictions as the model resolution progressively increases, the fully resolved part of the energy spectra must agree among runs of different $k_{t}$. This is achieved by controlling the forcing amplitude $\hat{A}$. Unfortunately, this has to be done ad experimentum, since, to our knowledge, no known formulae relate 
the forcing amplitude with the shape of the spectrum. The following choices of $\hat{A}$ are found to be appropriate following a series of fine-tuning tests: $\hat{A}=0.004$ for the large-scale forcing for all $k_{t}$; and $\hat{A}=0.005,0.006,0.007,0.008$ for the small-scale forcing for $k_{t}=256,512,1024,2048$ respectively. As shown in Figure 1, these particular choices also make the transition between the $k^{-3}$ and $k^{-\frac{5}{3}}$ ranges happen on the order of $k=100$, in agreement with the atmospheric energy spectrum observed by Nastrom and Gage (1985) where the spectral break sits at a length scale of about 400 kilometers. The spectra in Figure 1 are scaled by $k^{\frac{5}{3}}$ so that a perfect $k^{-\frac{5}{3}}$ range would appear as a horizontal line in the figure. It is apparent that the transition to a $k^{-\frac{5}{3}}$ spectrum is gradual, and is not even achieved in the highest-resolution run $\left(k_{t}=2048\right)$, although it is getting very close.

The two sets of perturbation experiments come in the form of identical twins - pairs of runs that differ only in the initial condition. The initial perturbations are introduced at a single wavenumber $k_{p}$ at a relative magnitude of $1 \%$, following the procedure of Leung et al. (2019). The first set explores the dependence of error growth properties on the scale $k_{p}$ of the initial error. There the model resolution is fixed to be the highest possible, i.e. $k_{t}=2048$, and perturbations are introduced at $k_{p}=128,256,512$ and 1024 . The second set explores the sensitivity of error growth to the model resolution by making $k_{t}$ variable. Model resolutions of $k_{t}=256,512,1024$ and 2048 are considered. $k_{p}$ is fixed relative to $k_{t}$ at $\frac{k_{p}}{k_{t}}=0.5$ so that the initial error is confined to a small scale yet unaffected by the forcing and dissipation. As such, the combination $\left(k_{t}, k_{p}\right)=(2048,1024)$ is included in both sets. For each combination of $\left(k_{t}, k_{p}\right)$, all results reported in this and the next section are averages over 5 independent realizations. 


\section{1) ERROR GROWTH AND ITS DEPENDENCE ON PERTURBATION SCALE}

Figure 2 shows the evolution of the error spectra for the different perturbation scales $k_{p}$ in the highest-resolution $\left(k_{t}=2048\right)$ model, where a substantial part of the unperturbed energy spectrum follows the $k^{-\frac{5}{3}}$ power-law reasonably well (Figure 1). The error spectra grow up-magnitude more or less uniformly across scales. As the mesoscale saturates, the error growth slows down, as indicated by the more closely packed spectra at later times. These observations are broadly consistent with the findings of Boffetta and Musacchio (2001), who simulated error growth in the inverse-cascade regime of $2 \mathrm{D}$ turbulence (i.e. a $k^{-\frac{5}{3}}$ control spectrum). They also agree with Judt (2018)'s study using a global convection-permitting NWP model.

Figure 2 also suggests that the dependence of error growth behavior on the perturbation scale $k_{p}$ is minimal, as manifested by the largely similar shape of the error spectra across the panels. This is in good agreement with Durran and Gingrich (2014). Decreasing the perturbation scale (increasing $k_{p}$ ) introduces a time-lag in saturating a given synoptic scale, but this lag decreases with the wavenumber and becomes negligible at the largest scales (not shown).

\section{2) DEPENDENCE ON MODEL RESOLUTION}

The results for the second set of experiments, in which the model resolution $k_{t}$ is variable, are shown in Figure 3. There is a qualitative difference between the error spectra of the low-resolution runs, where the $k^{-\frac{5}{3}}$ range is barely resolved (Figure 3(a,b)), and those of the high-resolution runs where the $k^{-\frac{5}{3}}$ range is resolved well (Figure 3(c,d)). Without a resolved mesoscale range, the error spectra peak at the synoptic scale (about $k=10$ ) throughout the growth process, following a short initial adjustment. This is consistent with previous studies (Rotunno and Snyder 2008; Durran and Gingrich 2014). In the presence of a mesoscale range, however, the error initially peaks at nearly 
the smallest resolved scale, i.e. towards the end of the $k^{-\frac{5}{3}}$ range, again echoing earlier studies (Lorenz 1969; Rotunno and Snyder 2008; Durran and Gingrich 2014). After the mesoscale error saturates, a separate peak in the synoptic scale begins to emerge in the error spectra, resembling the error growth paradigm under a $k^{-3}$ range. The same has been reported by Judt (2018) in the context of a high-resolution global NWP model.

Error spectra under a hybrid $k^{-3}$ and $k^{-\frac{5}{3}}$ spectrum thus show a stage-dependent peak and an up-magnitude growth at almost all stages. The analysis of the error growth behavior may be done more quantitatively by fitting the error growth to a parametric model and extracting information from the fitted parameters.

\section{Assessing the error growth rate using the parametric model of Žagar et al. (2017)}

\section{a. Description of the Žagar model}

The parametric model of Žagar et al. (2017) ('the Žagar model') approximates the evolution of some measure of the error energy by a scaled and translated hyperbolic tangent function

$$
E(t)=A \tanh (a t+b)+B
$$

where $t$ is the time since the initial perturbation, and $A>0, B \in \mathbb{R}, a>0$ and $b \in \mathbb{R}$ are parameters to be fitted. The measure of the error energy can be that at a particular wavenumber or a range of wavenumbers (which can be the total error energy), whether normalized by the saturation energy level or not. In this section, we apply the Žagar model on the normalized energy at individual wavenumbers, thus making equation (3) and its parameters functions of $k$ as well.

The $E$ given in equation (3) satisfies the autonomous differential equation

$$
\frac{\mathrm{d} E}{\mathrm{~d} t}=\frac{a}{A}\left(E_{\max }-E\right)\left(E-E_{\min }\right)
$$


where $E_{\max }:=A+B$ and $E_{\min }:=A-B$ are respectively the supremum and infimum attainable values of $E$ over all $t \in \mathbb{R}$. Equation (4) can be considered as an evolution equation for the error, with an initial condition of $E(t=0)=A \tanh (b)+B$. From this equation, one can see that the Žagar model is equivalent to the parametric error growth model of Dalcher and Kalnay (1987)

$$
\frac{\mathrm{d} E}{\mathrm{~d} t}=\left(\alpha_{1} E+\alpha_{2}\right)\left(1-\frac{E}{E_{\max }}\right)
$$

by noting that $\alpha_{1}=\frac{a}{A} E_{\max }$ and $\alpha_{2}=-\frac{a}{A} E_{\max } E_{\min }$ (Žagar et al. 2017). We focus on Žagar and her collaborators' formulation of the model here, as it provides an explicit expression for the parameterized error $E$ (equation (3)). If the evolution equation (4) or (5) were used instead, the parameters would then have to be fitted to the instantaneous growth rate $\frac{\mathrm{d} E}{\mathrm{~d} t}$, whose computation requires discretization and thus introduces inaccuracies.

\section{b. The fitting}

The fitting to equation (3) is carried out on Python's scipy . optimize package. Starting with an appropriate initial guess of the parameters $A, B, a$ and $b$, a least-squares minimization is performed by the Levenberg-Marquardt algorithm to compute the set of parameters that best approximates the evolution of the error.

As an illustration of the appropriateness of the hyperbolic tangent function in describing error growth, Figure 4 shows the evolution of the normalized error energy at a specific wavenumber and its best fit according to equation (3). The fit typically smoothens the error's fluctuations around the saturation level. Away from the saturation level, the fitting function matches the error almost perfectly. 
The contour plot in Figure 5(a) is obtained by repeating the fitting procedure independently for all wavenumbers. The corresponding plot for the raw, unfitted error is shown in Figure 5(b). It is evident that the fitting removes the noise and provides a cleaner signal to the error growth pattern.

\section{c. Inferring predictability from the parameters}

Parameter $a$ of equation (3) carries a mathematical interpretation. It controls the width of the hyperbolic tangent curve. By studying its dependence on $k, k_{t}$ and $k_{p}$, the predictability of the system can be inferred. To see this, let $E_{1}$ and $E_{2}$ be two arbitrary error energy levels with $E_{1}<E_{2}$, and $t_{1}$ and $t_{2}$ be the times when these energy levels are attained. If we write $F_{i}=\frac{E_{i}-B}{A}, i=1,2$, then equation (3) implies $a t_{i}+b=\tanh ^{-1}\left(F_{i}\right)$, so that

$$
t_{2}-t_{1}=\frac{1}{a}\left(\tanh ^{-1}\left(F_{2}\right)-\tanh ^{-1}\left(F_{1}\right)\right) .
$$

Since the hyperbolic tangent function is monotonically increasing, $\tanh ^{-1}\left(F_{2}\right)-\tanh ^{-1}\left(F_{1}\right)$ is always positive, meaning that a smaller $a$ always gives a larger (longer) $t_{2}-t_{1}$. As $a$ becomes larger, the curve narrows and thus suggests a more rapid error growth.

For the first set of experiments in which $k_{t}=2048$ and $k_{p}$ is variable, Figure 6 shows that $a$ increases with $k$ until the effects of the small-scale forcing become important. Hence, by the above argument, the error grows faster as the spatial scale decreases. This is particularly apparent in the $k^{-\frac{5}{3}}$ mesoscale range, where the slope $\frac{\mathrm{d} a}{\mathrm{~d}(\log k)}$ increases. This is a hallmark of inherently finite predictability, and reinforces the agreement with Judt (2018)'s earlier study using a more sophisticated NWP model.

It is interesting to see that $a$ increases more rapidly in the mesoscale when $k_{p}$ is smaller. In other words, error growth in the mesoscale is faster when the perturbation is applied at a larger scale. 
This may be attributable to the fast transfer of larger-scale errors into the smaller scales (Durran and Gingrich 2014).

Figure 7 shows $a(k)$ for the second set of experiments, in which $\frac{k_{p}}{k_{t}}$ is fixed at 0.5 . It is quite remarkable that the values of $a$ for the different resolutions are broadly consistent (as long as they lie outside the forcing ranges), meaning that the error growth at a given scale is not substantially altered by pushing the model to a higher resolution. Having said that, the distinctively changing slope $\frac{\mathrm{d} a}{\mathrm{~d}(\log k)}$ for the highest-resolution run $k_{t}=2048$ (the same magenta curve as in Figure 6) is not seen when $k_{t}$ is smaller.

The heuristic dimensional argument for homogeneous and isotropic turbulence (Lilly 1990) implies that the parameter $a$ should scale as $\left[k^{3} E(k)\right]^{\frac{1}{2}}$, since it carries the physical dimension of inverse time. Accordingly, $a$ should be constant in $k$ if the energy spectrum is $k^{-3}$, and should scale as $k^{\frac{2}{3}}$ if $E(k) \sim k^{-\frac{5}{3}}$. However, Figure 7 suggests that $a$ scales with $k$ logarithmically in the large scales. Into the small scales of the highest-resolution runs, a polynomial scaling seems to emerge, but in any case it falls well short of $k^{\frac{2}{3}}$ which demands a more-than-fourfold increase in $a$ for every decade of wavenumbers. Hence, the observed behavior of $a$ remains in an intermediate, non-asymptotic regime, as might be expected under a hybrid $k^{-3}$ and $k^{-\frac{5}{3}}$ energy spectrum.

\section{Exploring the asymptotic behavior using Lorenz's model}

It is of interest to investigate the characteristics of error growth under the hybrid spectrum in the infinite-resolution limit. To achieve this, a much higher-resolution model is needed to reasonably serve as a proxy for the infinite-resolution case. The primitive model of Lorenz (1969) is a good candidate for this purpose, as its computational inexpensiveness enables running of ultra-highresolution simulations. 
Lorenz's model is based on the dimensionless 2D barotropic vorticity equation (1) but without forcing and dissipation $(f=d=0)$. This is equivalent to the vorticity form of the incompressible 2D Euler equations. Forcing and dissipation are instead implicit in the nature of the assumed background energy spectrum. Expanding its linearized error equation in a Fourier basis, making certain simplifying assumptions (e.g. turbulence closure) and discretizing it, the model reduces to a system of linear ordinary differential equations

$$
\frac{\mathrm{d}^{2}}{\mathrm{~d} t^{2}} Z=C Z
$$

where $Z$ is a vector of error energies at different scales (each scale $K$ collectively represents wavenumbers $k=2^{K-1}$ to $k=2^{K}$ ), and $C$ is a matrix of constant coefficients. Given the resolution $K_{\max }$ of the model, the entries of $C$ only depend on the energy spectrum of the unperturbed flow, which is specified a priori by the user. Further details on the derivation of the model, including the computation of $C$, are available in Lorenz (1969), Rotunno and Snyder (2008), and Leung et al. (2019). For a given initial condition of $Z$ and its first time-derivative, the model is solved analytically following the procedure of Leung et al. (2019). When the error at a particular scale saturates, the error energy at that scale ceases to be a prognostic variable of equation (7), but its effects on the remaining scales via the matrix $C$ are retained in the form of an inhomogeneous forcing while the time-integration continues.

\section{a. Reproducing the DNS results}

We first demonstrate that Lorenz's model is able to capture the essential aspects of error growth observed in the direct numerical simulations (DNS) of Sections 2 and 3. Specifically, we show this for the set of experiments in which $\frac{k_{p}}{k_{t}}$ is fixed (cf. Figure 3). To compute the matrix $C$ and hence run the model, the background energy spectra at the final time $(t=150)$ of the identical- 
twin simulations in Section 2 are recycled. For each $\left(k_{t}, k_{p}\right)$ pair, a single background spectrum is formed by averaging the 5 independent realizations. Next, the spikes induced by the forcing are removed, with the energy spectral densities at the forced wavenumbers replaced by interpolation of the densities at the neighbouring wavenumbers outside the forced range (the interpolation is linear in log-log space in order to respect the power-law nature of the spectrum). The resulting spectrum is then discretized into the scales $K$, with minimum $K_{\min }=1$ and maximum $K_{\max }=\log _{2} k_{t}=8,9$, 10 and 11 respectively.

The model (7), with $C$ computed from the discretized spectrum, is solved for one-half of the initial error drawn from the respective DNS. (The factor of one-half is due to the definition of the error in Lorenz's model based on turbulence closure concepts, which makes the re-defined error saturate at the control energy spectrum rather than twice its level.) The initial condition for $\frac{\mathrm{d} Z}{\mathrm{~d} t}$ is set to be zero for all $K$, as it will be for the remainder of the article.

Figure 8 shows the parameter $a$ of the Žagar model as a function of $K$. Compared to the growth rates for the DNS (Figure 7), the single most distinctive feature - that $a$ generally increases as $k$ or $K$ increases, albeit much slower than the heuristic scaling would suggest - is captured in Lorenz's model. In other words, Lorenz's model is able to reproduce the moderate quickening of error growth in the mesoscale, though not to the same extent as in the DNS themselves (the values of $a$ in the mesoscale range in Figure 8 are generally smaller than in Figure 7 by a factor of two). Lorenz's model also captures the suppression of error growth at intermediate scales in the higher-resolution simulations, as seen in Figure 7.

It should be noted that Lorenz's model is, in some cases, known to produce unrealistically oscillatory error behavior at small times (Lorenz 1969). This includes the emergence of transient negative error energy values, which is in no way excluded by the mathematical formulation of the model. Indeed, it is a known shortcoming of the quasi-normal turbulence closure which Lorenz 
used in deriving his model (Orszag 1970). Nevertheless, qualitatively speaking, the erratic behavior amounts to nothing more than a time-delay in error growth. Therefore, it does not affect our concerned parameter $a$ of the Žagar model, since the time-delay is represented in the parameter $b$.

\section{b. Error growth in the infinite-resolution limit}

Having demonstrated the ability of Lorenz's model to reproduce the basic features of error growth, we turn our focus to the ultra-high-resolution case, $K_{\max }=21$. Physically, it corresponds to a minimum wavelength of about 19 metres on Earth, well beyond the resolution of today's NWP models.

The discretized background spectrum used for the $K_{\max }=11$ simulation above is extended to $K_{\max }=21$, assuming a pure $k^{-\frac{5}{3}}$ range at these smaller scales. In other words, for all integers $K \in[11,21)$

$$
\frac{X(K+1)}{X(K)}=2^{-\frac{2}{3}}
$$

The scaling $2^{-\frac{2}{3} K}=k^{-\frac{2}{3}}=k^{-\frac{5}{3}+1}$ is the energy integrated over a unit logarithm of wavenumbers when the energy spectral density scales as $k^{-\frac{5}{3}}$.

Figure 9(a) illustrates the growth of a small-scale error under this hybrid background spectrum extended to $K_{\max }=21$. The error spectrum exhibits a fairly sharp peak at all lead times, in contrast with the lower-resolution case (e.g. Figure 3(d)) where the peak is much broader. Figure 9(b) shows the same but for a single $k^{-\frac{5}{3}}$ range, defined by

$$
X(K)=2^{-\frac{2}{3} K}-2^{-K},
$$

yet normalized to such a level that the magnitude of the mesoscale part of the spectrum agrees with that in Figure 9(a). The second term of equation (9) represents a correction to $k^{-\frac{5}{3}}$ whose effect is most significant in the large scales, where the shape of the spectrum departs from the 
power-law. The formulation of this spectrum is therefore identical to Lorenz (1969), save the normalization, and enables a direct comparison with Figure 9(a) for examining the effects of an additional $k^{-3}$ range in the synoptic scale (it should be noted that in this way the hybrid spectrum is more energetic in absolute terms). There is a very close agreement between the nature of the mesoscale error growth in Figure 9(a) and in Figure 9(b). It seems plausible, then, to suggest that the error under the hybrid spectrum asymptotically behaves as the error under a single $k^{-\frac{5}{3}}$ range, and that the presence of the $k^{-3}$ range does not affect the fast error growth at the smallest scales. This comparison also suggests that $K_{\max }=21$ is sufficient to be considered a proxy for the infinite-resolution limit.

This can be expressed in more quantitative terms by considering the parameter $a$ of the Žagar model (Figure 10(a)). For $K_{\max }=21$ (black solid curve), $a$ grows exponentially beyond $K=11$. This growth is very similar in simulations at intermediate resolutions, confirming that our results have converged in this respect. Indeed, the growth is even faster than the theoretically expected scaling of $k^{\frac{2}{3}}=2^{\frac{2}{3} K}$ for a $k^{-\frac{5}{3}}$ spectrum. The implication here is that it is necessary to fully resolve $K=11$ (19.5 to 39.1 kilometers on Earth) for the model to pick up the fast error growth pertaining to the $k^{-\frac{5}{3}}$ range, despite it being more than a decade of wavenumbers beyond the spectral break between the $k^{-3}$ and $k^{-\frac{5}{3}}$ ranges. Moreover, the results suggest that the synoptic-scale $k^{-3}$ acts to slow down error growth in the first decade of the mesoscale. This is also supported by $a(K)$ 's approximate proportionality to $2^{\frac{2}{3} K}$ for all $K$ in the single-range $k^{-\frac{5}{3}}$ spectrum (not shown).

We can update Lorenz (1969)'s estimate of the predictability horizon using this hybrid spectrum. Table 1 lists the error saturation time for each $K$, dimensionalized using his estimate of the root-mean-square wind speed in the upper troposphere (17.1824 meters per second). Generally speaking, a change in the magnitude of the initial error at the smallest scale would shift the predictability horizons across the whole table by a near-constant amount (not shown), so that the 
ranges of predictability at the large scales are relatively more robust than at the small scales. The predictability limit for the planetary scale is estimated to be about 15 to 20 days, in line with recent estimates using more sophisticated models (Buizza and Leutbecher 2015; Judt 2018; Zhang et al. 2019).

\section{Other initial error profiles}

In Section 4, we focused on cases where the initial error is concentrated at the smallest available scale, thereby approximating an infinitesimally small-scale error. This is analogous to Lorenz (1969)'s well-known Experiment A. Initial error spectra in realistic weather forecasts are, however, very different. To explore the sensitivity of the error growth behavior to the initial error spectrum, Lorenz performed the lesser-known Experiments B and C. In his Experiment B, the initial error was confined to the largest-available scale, whereas Experiment $\mathrm{C}$ was initialized with a fixed fraction of the control energy spectrum across all scales. He concluded that the predictability horizon at the planetary scale is barely dependent on the initial error spectrum. Durran and Gingrich (2014) expanded on Lorenz's results to show that, despite the insensitivity of the predictability horizon, the error spectra in Experiments B and C grow somewhat differently from Experiment A (their Figures 2(a) and 3). They also demonstrated that additional small-scale 'butterflies' are practically irrelevant to the error growth pattern when the initial error spectrum has a non-negligible contribution from the large scales.

Here, Durran and Gingrich (2014)'s experiments are repeated for the hybrid background spectrum with $K_{\max }=21$. The growth of the error spectrum is shown in Figure 11. In Figure 11(a), the initial error is confined to the largest scale, whereas in Figure 11(b) the initial error is distributed across all scales in a uniform manner relative to the control spectrum. The error spectra have 
similar shapes beyond the initial time, and both figures conform nicely to Durran and Gingrich (2014)'s result.

The Žagar error-growth parameter $a(K)$ for both alternative initial conditions is seen to follow the same general pattern as the case in which the initial error is at the smallest scale (Figure 10(b)). In particular, the exponential growth of $a$ from $K=11$ and the sluggish variation at smaller $K$ still hold. Indeed, differences in $a(K)$ across the three cases are practically invisible for all $K \leq 14$. Beyond $K=14$, the curves for the large-scale and proportional initial errors remain nearly identical to each other but are distinct from the curve for the small-scale initial error by a small margin. The overall excellent agreement across the three initial error profiles therefore extends Durran and Gingrich (2014)'s conclusion - that "the loss of predictability generated by initial errors of small but fixed absolute magnitude is essentially independent of their spatial scale" - to the hybrid spectrum. Yet the comparison also shows that the inferences obtained from our version of Lorenz's Experiment A are robust to different initial error distributions.

\section{Summary and conclusions}

Building on Judt (2018)'s study which shows that model-world errors in a convection-permitting global NWP model demonstrate mixed characteristics of error growth under a hybrid $k^{-3}$ and $k^{-\frac{5}{3}}$ spectrum, we examined in this paper the sensitivity of error growth properties to the model resolution or, in other words, to the extent to which the $k^{-\frac{5}{3}}$ mesoscale range is explicitly resolved. This was done in a 2D barotropic vorticity model. The use of simple models for casting light on error growth and predictability properties in the real world is justified as long as the Nastrom-Gage hybrid $k^{-3}-k^{-\frac{5}{3}}$ energy spectrum is well-modelled, since these properties are largely determined by the shape of the spectrum (Rotunno and Snyder 2008). 
Results from identical-twin perturbation experiments with the 2D barotropic vorticity model at a range of resolutions (Section 2) show that a stage-dependent peak in the error energy spectrum begins to emerge as the model resolution increases from $k_{t}=256$ (where there is essentially no room for the $k^{-\frac{5}{3}}$ range) to $k_{t}=2048$ (where the mesoscale range is substantially resolved). Under the hybrid spectrum, the error spectrum initially peaks at the small scales until the $k^{-\frac{5}{3}}$ range becomes saturated, then a synoptic-scale peak characteristic of error growth under a $k^{-3}$ spectrum starts to appear. These observations echo Judt (2018)'s findings, and confirm that the 2D barotropic vorticity equation can mimic the essential aspects of this process.

The dependence of the error growth rate on spatial scale is used to quantitatively characterize the predictability of the system. A measure of this rate is the parameter $a$ of the parametric error growth model of Žagar et al. (2017) (Section 3). By fitting the error energy data obtained from the perturbation experiments to this parametric model, it is shown that the error indeed grows faster as the spatial scale decreases, thereby providing a hint of limited predictability. This is particularly evident in the $k^{-\frac{5}{3}}$ range. However, the increase in the growth rate as the spatial scale decreases falls well short of the theoretical estimate, thus indicating that the error behavior has not reached the asymptotic regime pertaining to this mesoscale range.

The model of Lorenz (1969), which is also based on the 2D barotropic vorticity equation, is used to investigate the asymptotic behavior (Section 4). At a modest computational cost, Lorenz's model successfully captures the important characteristics of error growth, thus enabling ultrahigh-resolution simulations for estimating growth patterns in the continuum. It is found that under the hybrid spectrum, the fast upscale cascade of error energy characteristic of limited predictability becomes unambiguously visible only beyond $k=2048=2^{11}$ (19.5 kilometers), more than a decade of wavenumbers beyond the spectral break between the synoptic-scale and mesoscale ranges. Until then, the synoptic-scale range suppresses mesoscale error growth. 
Applying these results to NWP would mean that models have to fully resolve the dynamics at the scale of the typical grid resolution of today's global ensembles ( $\sim 20$ kilometers $)$ in order for the fast mesoscale uncertainty growth to be accurately captured within the model. Based on Skamarock (2004), this would suggest a grid resolution 7 times finer than typical of today, i.e. on the order of a few kilometers, after accounting for the need for a dissipation range. Pushing NWP models to such a resolution can be anticipated to provide a more realistic description of smallscale error growth and thus of the uncertainty in the forecast, even when the initial errors are not confined to the smallest scales (Section 5). Yet, we recognize that developing stochastic parameterizations for processes on the $O(1)$-kilometer scale (e.g. cloud processes) may also achieve the same purpose. It should also be noted that realistic initial error profiles have typically far greater amplitudes than those considered in the present study, whose focus is on predictability properties in the limiting case.

Judt (2020) suggests that the canonical hybrid $k^{-3}-k^{-\frac{5}{3}}$ spectrum, which has been assumed here throughout, is restricted to the mid-latitude upper troposphere only. The applicability of these results to other parts of the atmosphere, or indeed to the atmosphere as a whole, remains a topic of further research.

Acknowledgments. Tsz Yan Leung is supported through a PhD scholarship awarded by the Engineering and Physical Sciences Research Council grant EP/L016613/1 'EPSRC Centre for Doctoral Training in the Mathematics of Planet Earth at Imperial College London and the University of Reading', with additional funding support from the European Research Council Advanced Grant 'Understanding the Atmospheric Circulation Response to Climate Change' (ACRCC), Project 339390, under Theodore G. Shepherd as the Principal Investigator. The work of Sebastian Reich has been partially funded by Deutsche Forschungsgemeinschaft (DFG, German Science Founda- 
tion) - SFB 1114/2 235221301. The authors thank Richard Scott for providing his code for modification for the numerical simulations in Section 2, which would have not been possible without further support from high-performance computing resources at the European Centre for Mediumrange Weather Forecasts. Chris Snyder, Falko Judt, and an anonymous reviewer are thanked for their useful comments.

\section{References}

Boer, G. J., and T. G. Shepherd, 1983: Large-scale two-dimensional turbulence in the atmosphere.

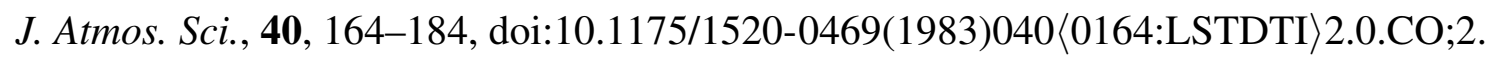

Boffetta, G., and S. Musacchio, 2001: Predictability of the inverse energy cascade in 2d turbulence. Physics of Fluids, 13, 1060-1061, doi:10.1063/1.1350877.

Buizza, R., and M. Leutbecher, 2015: The forecast skill horizon. Quart. J. Roy. Meteor. Soc., 141, 3366-3382, doi:10.1002/qj.2619.

Dalcher, A., and E. Kalnay, 1987: Error growth and predictability in operational ecmwf forecasts. Tellus, 39A, 474-491, doi:10.1111/j.1600-0870.1987.tb00322.x.

Durran, D. R., and M. Gingrich, 2014: Atmospheric predictability: why butterflies are not of practical importance. J. Atmos. Sci., 71, 2476-2488, doi:10.1175/JAS-D-14-0007.1.

Judt, F., 2018: Insights into atmospheric predictability through global convection-permitting model simulations. J. Atmos. Sci., 75, 1477-1497, doi:10.1175/JAS-D-17-0343.1.

Judt, F., 2020: Atmospheric predictability of the tropics, middle latitudes, and polar regions explored through global storm-resolving simulations. J. Atmos. Sci., 77, 257-276, doi:10.1175/ JAS-D-19-0116.1. 
Leung, T. Y., M. Leutbecher, S. Reich, and T. G. Shepherd, 2019: Atmospheric predictability: revisiting the inherent finite-time barrier. J. Atmos. Sci., 76, 3883-3892, doi:10.1175/ JAS-D-19-0057.1.

Lilly, D. K., 1990: Numerical prediction of thunderstorms - has its time come? Quart. J. Roy. Meteor. Soc., 116, 779-798, doi:10.1002/qj.49711649402.

Lorenz, E. N., 1969: The predictability of a flow which possesses many scales of motion. Tellus, 21, 289-307, doi:10.3402/tellusa.v21i3.10086.

Maltrud, M. E., and G. K. Vallis, 1991: Energy spectra and coherent structures in forced two-dimensional and beta-plane turbulence. J. Fluid Mech., 228, 321-342, doi:10.10.1017/ S0022112091002720.

Nastrom, G. D., and K. S. Gage, 1985: A climatology of atmospheric wavenumber spectra of wind and temperature observed by commercial aircraft. J. Atmos. Sci., 42, 950-960, doi:10. 1175/1520-0469(1985)042〈0950:ACOAWS $\rangle 2.0 . C O ; 2$.

Orszag, S. A., 1970: Analytical theories of turbulence. J. Fluid Mech., 41, 363-386, doi:10.1017/ S00221120700006421.

Rotunno, R., and C. Snyder, 2008: A generalization of Lorenz's model for the predictability of flows with many scales of motion. J. Atmos. Sci., 65, 1063-1076, doi:10.1175/2007JAS2449.1.

Skamarock, W. C., 2004: Evaluating mesoscale NWP models using kinetic energy spectra. Mon. Wea. Rev., 132, 3019-3032, doi:10.1175/MWR2830.1.

Sun, Y. Q., and F. Zhang, 2016: Intrinsic versus practical limits of atmospheric predictability and the significance of the butterfly effect. J. Atmos. Sci., 73, 1419-1438, doi:10.1175/ JAS-D-15-0142.1. 
Žagar, N., M. Horvat, Ž. Zaplotnik, and L. Magnusson, 2017: Scale-dependent estimates of the growth of forecast uncertainties in a global prediction system. Tellus A: Dynamic Meteorology and Oceanography, 69:1, 1287492 , doi:10.1080/16000870.2017.1287492.

Zhang, F., Y. Q. Sun, L. Magnusson, R. Buizza, S.-J. Lin, J.-H. Chen, and K. Emanuel, 2019: What is the predictability limit of midlatitude weather? J. Atmos. Sci., 76, 1077-1091, doi: 10.1175/JAS-D-18-0269.1. 
472 LIST OF TABLES

Table 1. Dimensionalized error saturation times (i.e. predictability horizons) for various length scales $K$, computed using Lorenz (1969)'s model for 21 scales, and the same control energy spectrum and initial error as Figure 9(a). 
TABLE 1. Dimensionalized error saturation times (i.e. predictability horizons) for various length scales $K$, computed using Lorenz (1969)'s model for 21 scales, and the same control energy spectrum and initial error as

478 Figure 9(a).

\begin{tabular}{|c|c|c|}
\hline$K$ & Length scale & Predictability horizon \\
\hline 1 & $20000-40000 \mathrm{~km}$ & 20.1 days \\
\hline 2 & $10000-20000 \mathrm{~km}$ & 15.8 days \\
\hline 3 & $5000-10000 \mathrm{~km}$ & 12.6 days \\
\hline 4 & $2500-5000 \mathrm{~km}$ & 10.3 days \\
\hline 5 & $1250-2500 \mathrm{~km}$ & 8.74 days \\
\hline 6 & $625-1250 \mathrm{~km}$ & 6.46 days \\
\hline 7 & $313-625 \mathrm{~km}$ & 5.31 days \\
\hline 8 & $156-313 \mathrm{~km}$ & 4.30 days \\
\hline 9 & $78.1-156 \mathrm{~km}$ & 3.53 days \\
\hline 10 & $39.1-78.1 \mathrm{~km}$ & 2.52 days \\
\hline 11 & $19.5-39.1 \mathrm{~km}$ & 1.24 days \\
\hline 12 & $9.77-19.5 \mathrm{~km}$ & 20.4 hours \\
\hline 13 & $4.88-9.77 \mathrm{~km}$ & 10.8 hours \\
\hline 14 & $2.44-4.88 \mathrm{~km}$ & 7.19 hours \\
\hline 15 & $1.22-2.44 \mathrm{~km}$ & 4.89 hours \\
\hline 16 & $610 \mathrm{~m}-1.22 \mathrm{~km}$ & 2.62 hours \\
\hline 17 & $305-610 m$ & 1.88 hours \\
\hline 18 & $153-305 m$ & 1.35 hours \\
\hline 19 & $76.2-153 \mathrm{~m}$ & 58.0 minutes \\
\hline 20 & $38.1-76.2 \mathrm{~m}$ & 47.0 minutes \\
\hline 21 & $19.1-38.1 \mathrm{~m}$ & 41.1 minutes \\
\hline
\end{tabular}




\section{LIST OF FIGURES}

Fig. 1. Background energy spectra, scaled by a factor of $k^{\frac{5}{3}}$, for model resolutions $k_{t}=256$ (magenta), 512 (green), 1024 (blue) and 2048 (red). The black curve shows a logarithmically corrected $k^{-3}$ reference spectrum $E(k) \sim k^{-3}\left[\log \left(\frac{k}{15}\right)\right]^{-\frac{1}{3}}$, again scaled by a factor of $k^{\frac{5}{3}}$. The spectra are averaged over 5 independent realizations that differ in the random seed. The prominent peaks are associated with the mesoscale forcing, while the steep drop-off at the smallest scales is associated with the hyper-viscosity.

Fig. 2. Evolution of error energy spectra (blue, from bottom to top within each panel) for identicaltwin experiments with $k_{t}=2048$ and $k_{p}=$ (a) 128 , (b) 256, (c) 512 and (d) 1024 . The error spectra are plotted at equal time intervals. The blue dots indicate the scale $\left(k_{p}\right)$ and magnitude of the initial perturbations, and the red curves indicate the energy spectra of the unperturbed runs (scaled by a factor of two). All results presented here are averages over 5 independent realizations.

Fig. 3. As in Figure 2, but for $k_{t}=$ (a) 256, (b) 512, (c) 1024 and (d) 2048, and $k_{p}=\frac{1}{2} k_{t}$. Note that (d) is identical to Figure 2(d).

Fig. 4. Growth of the error energy at $k=70$ in the $\left(k_{t}, k_{p}\right)=(2048,1024)$ simulation, normalized by twice the background energy at the same wavenumber, in red. The blue curve shows the best fit of the red curve to the Žagar model according to equation (3). The data are averaged over 5 independent realizations before the fitting is performed.

Fig. 5. The growth of the (a) fitted and (b) raw errors as functions of the wavenumber, for the same simulations as in Figure 4. The colors and contours indicate the normalized error energy level.

Fig. 6. Parameter $a$ of the Žagar model, fitted to the normalized error energy at individual wavenumbers according to equation (3), as a function of the wavenumber, for perturbation experiments of various $k_{p}$ for the highest-resolution model $k_{t}=2048$. The data are averaged over 5 independent realizations before the fitting is performed. Note that the vertical axis is linear and not logarithmic.

Fig. 7. As in Figure 6, but for combinations of $\left(k_{t}, k_{p}\right)$ such that $k_{p}=\frac{1}{2} k_{t}$.

Fig. 8. As in Figure 7, but for the Lorenz (1969) model.

Fig. 9. (a) Evolution of the error energy spectrum (blue and magenta, from bottom to top) in the Lorenz (1969) model under the control energy spectrum (red) recovered from the $\left(k_{t}, k_{p}\right)=$ $(2048,1024)$ simulations in Section 2 (with modifications, details of which are given in the text) and extended to $K_{\max }=21$ via equation (8), and an initial condition of $Z\left(K_{\max }\right)=$ $5 \times 10^{-7} \times \sum_{L=1}^{K_{\max }} X(L)$ and $Z(K)=0$ for all other $K$. (b) As in (a), but for a single-range $k^{-\frac{5}{3}}$ control energy spectrum according to equation (9) yet normalized to such a level that the magnitude of the mesoscale part of the spectrum coincides with (a). The error spectra are plotted in blue at equal time-intervals of $\Delta t=3$ up to $t=60$, and in magenta at intervals of $\Delta t=30$ thereafter. The vertical axes show the equivalent energy spectral density $2^{-K} Z(K)$, a function that smoothly distributes $Z(K)$ which would have been a density in $k$ had $K$ been a continuous variable.

Fig. 10. (a) As in Figure 8, but for $K_{\max }=11$ (cyan), 13 (red), 15 (green), 17 (blue), 19 (magenta) and 21 (black), and an initial condition of $Z\left(K_{\max }\right)=5 \times 10^{-7} \times \sum_{L=1}^{K_{\max }} X(L)$ and $Z(K)=0$ 
for all other $K$. (b) shows the same black curve for the $K_{\max }=21$ simulation as (a), and additionally for cases where the initial condition of the same magnitude is moved to $K=$ 1 (red) or redistributed as a uniform fraction of the background spectrum (blue, which is essentially indistinguishable from the red). The vertical axes are logarithmic and the dashed

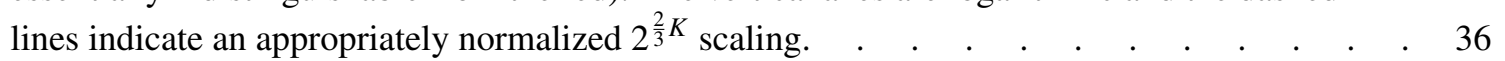

Fig. 11. As in Figure 9(a), but for the following initial conditions for $Z$ : (a) $Z(1)=5 \times 10^{-7} \times$ $\sum_{L=1}^{K_{\max }} X(L)$ and $Z(K)=0$ for all other $K$; (b) $Z(K)=5 \times 10^{-7} \times X(K)$ for all $K$. 


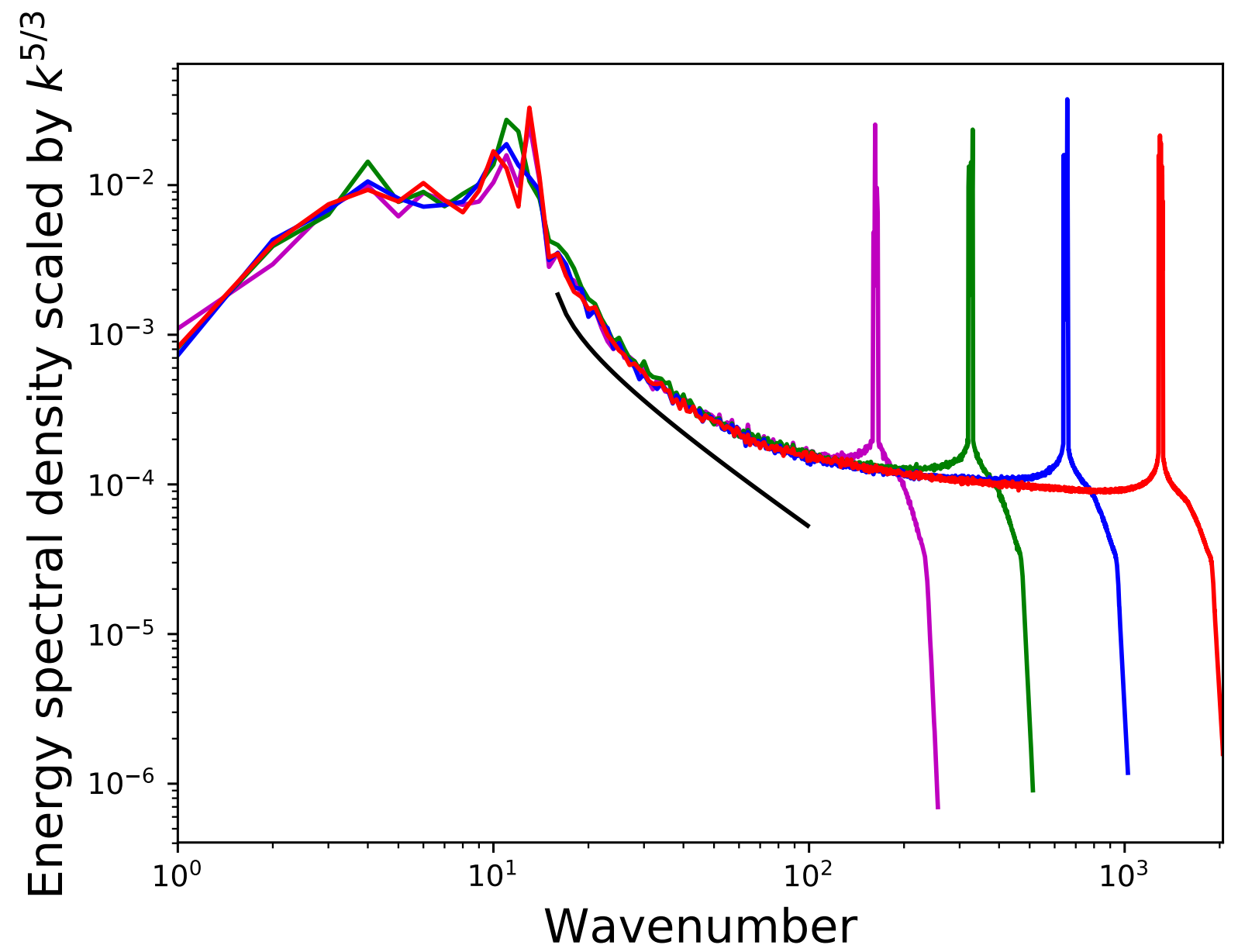

FIG. 1. Background energy spectra, scaled by a factor of $k^{\frac{5}{3}}$, for model resolutions $k_{t}=256$ (magenta), 512

(green), 1024 (blue) and 2048 (red). The black curve shows a logarithmically corrected $k^{-3}$ reference spectrum $E(k) \sim k^{-3}\left[\log \left(\frac{k}{15}\right)\right]^{-\frac{1}{3}}$, again scaled by a factor of $k^{\frac{5}{3}}$. The spectra are averaged over 5 independent realizations that differ in the random seed. The prominent peaks are associated with the mesoscale forcing, while the steep drop-off at the smallest scales is associated with the hyper-viscosity. 

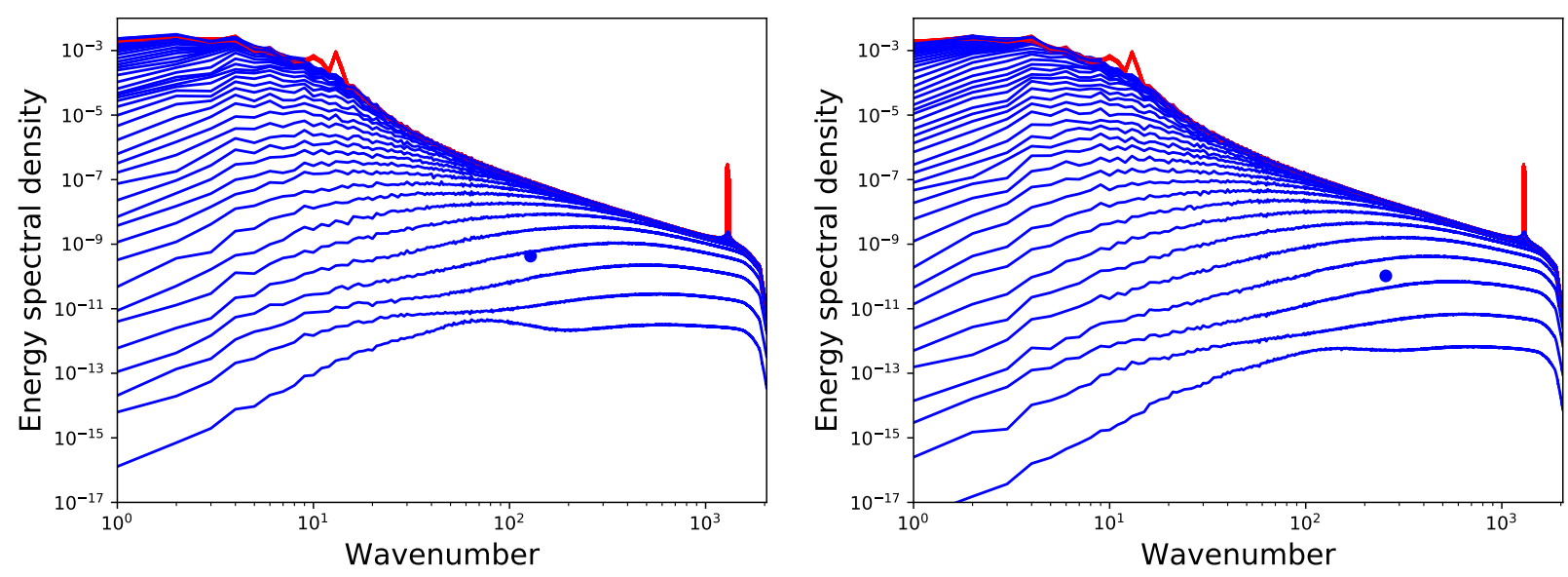

(a)

(b)
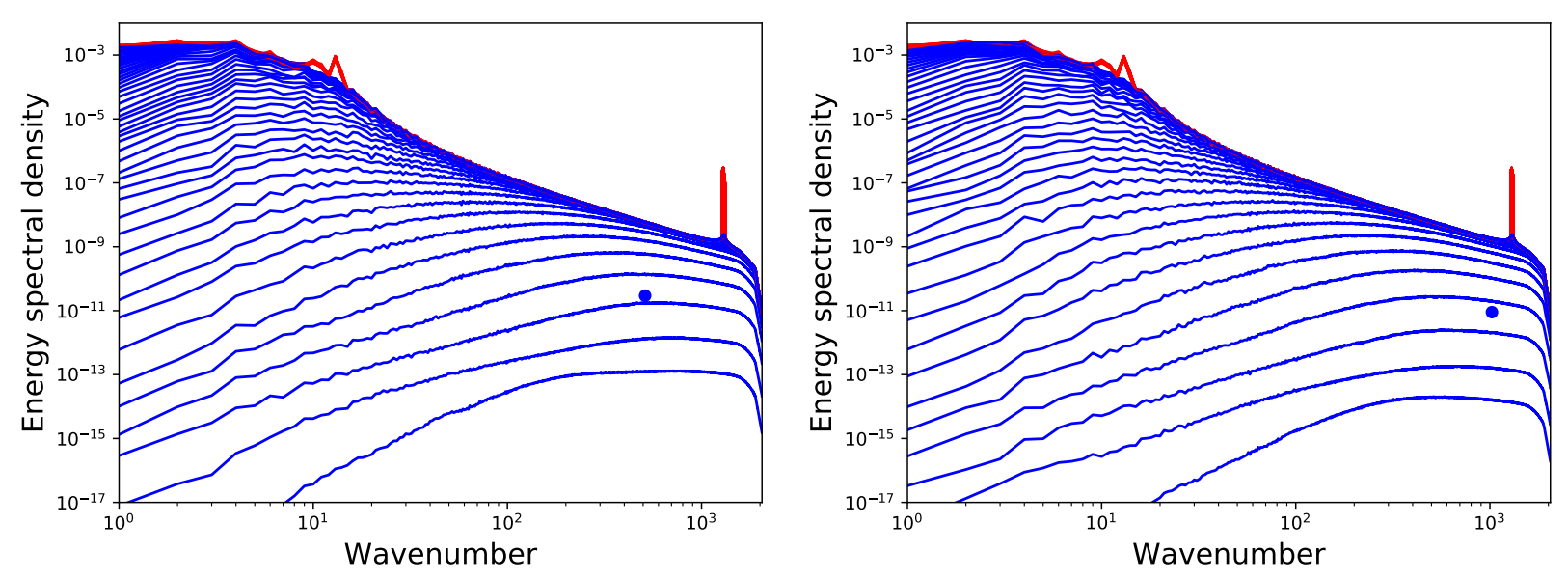

(c)

(d)

FIG. 2. Evolution of error energy spectra (blue, from bottom to top within each panel) for identical-twin experiments with $k_{t}=2048$ and $k_{p}=$ (a) 128, (b) 256, (c) 512 and (d) 1024. The error spectra are plotted at equal time intervals. The blue dots indicate the scale $\left(k_{p}\right)$ and magnitude of the initial perturbations, and the red curves indicate the energy spectra of the unperturbed runs (scaled by a factor of two). All results presented here are averages over 5 independent realizations. 

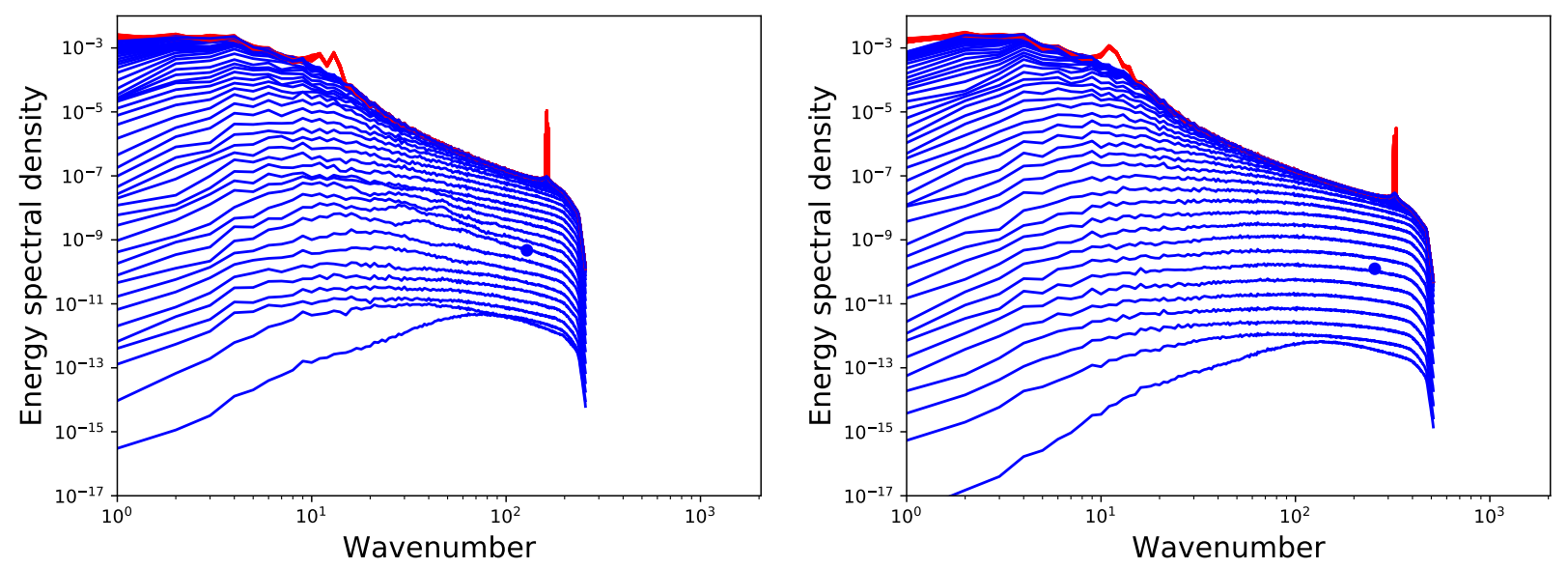

(a)

(b)
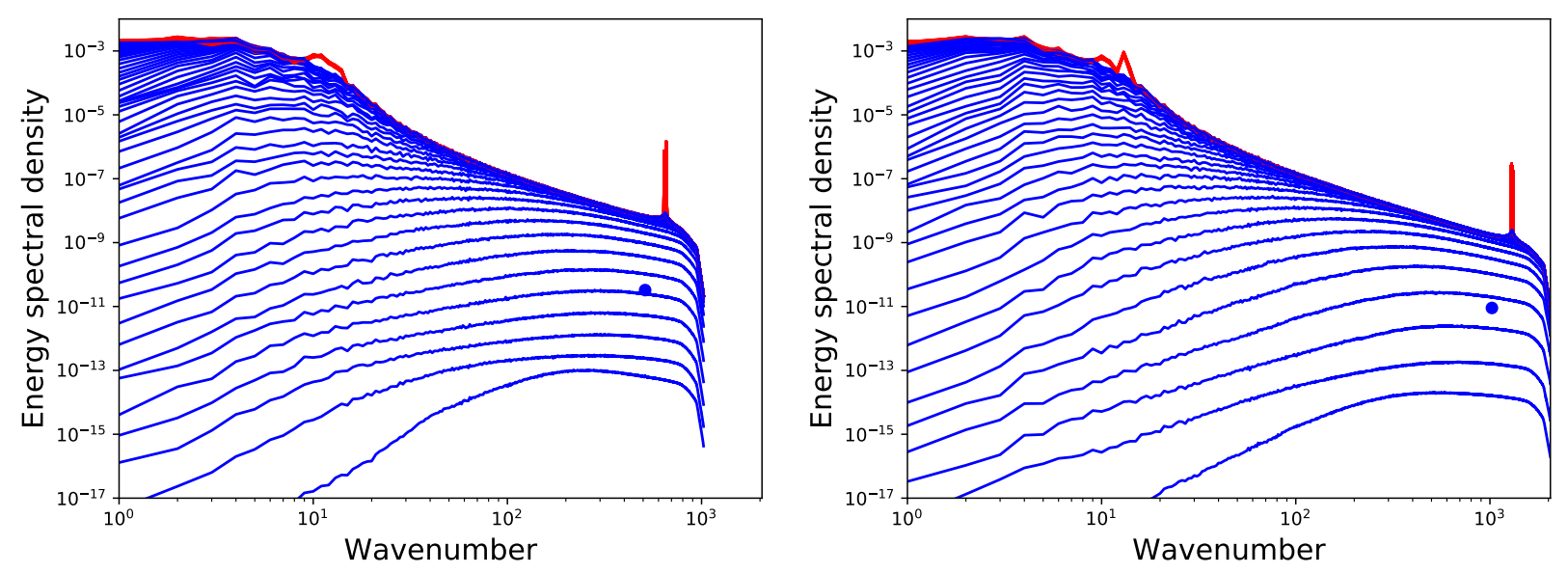

(c)

FIG. 3. As in Figure 2, but for $k_{t}=$ (a) 256, (b) 512, (c) 1024 and (d) 2048, and $k_{p}=\frac{1}{2} k_{t}$. Note that (d) is identical to Figure 2(d). 


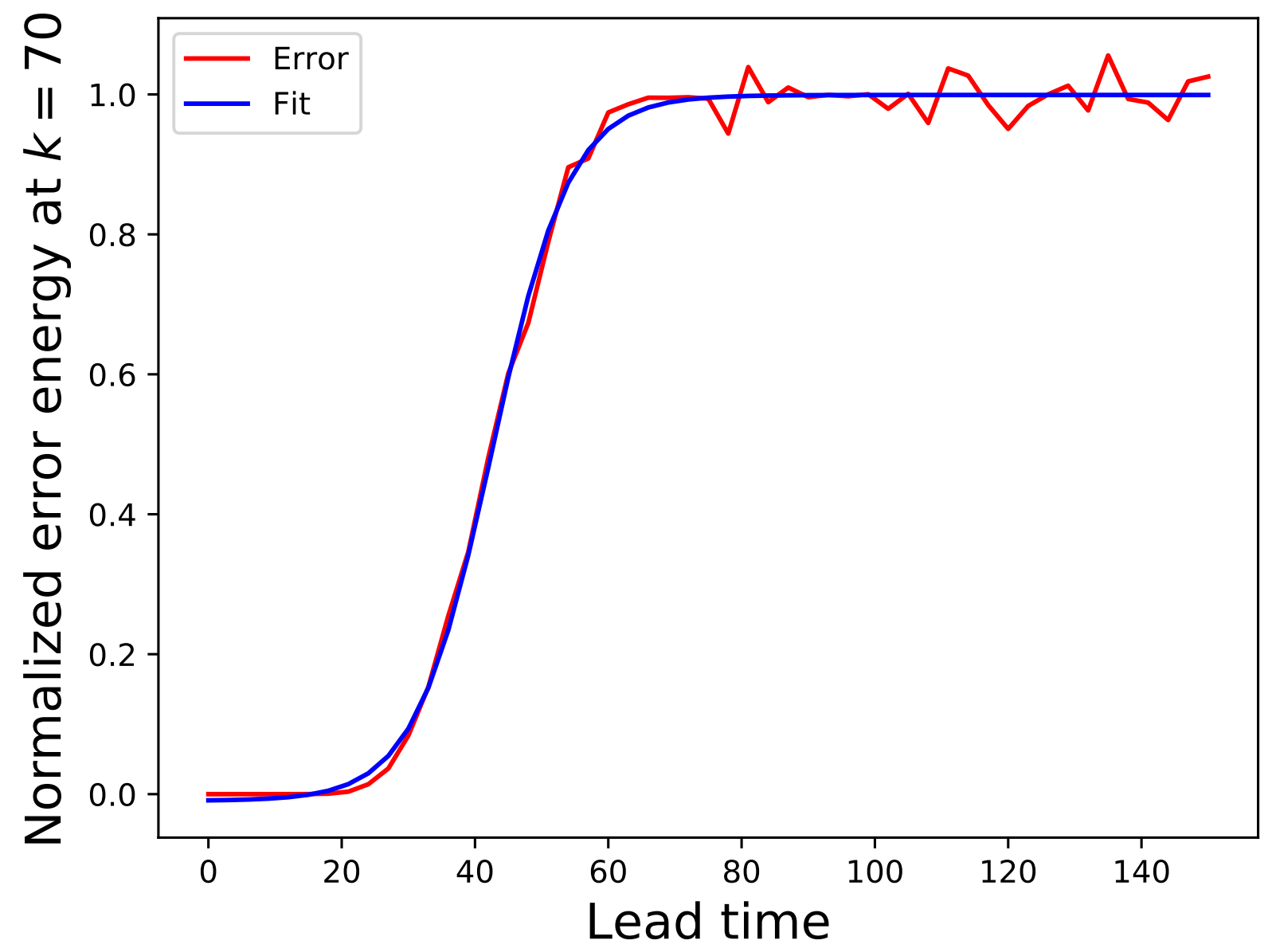

FIG. 4. Growth of the error energy at $k=70$ in the $\left(k_{t}, k_{p}\right)=(2048,1024)$ simulation, normalized by twice the background energy at the same wavenumber, in red. The blue curve shows the best fit of the red curve to the 542 Žagar model according to equation (3). The data are averaged over 5 independent realizations before the fitting is performed. 


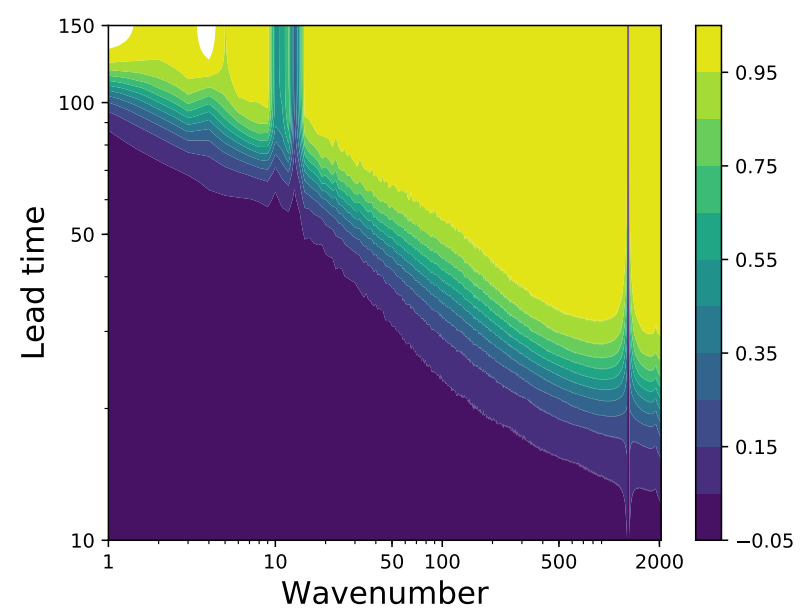

(a)

FIG. 5. The growth of the (a) fitted and (b) raw errors as functions of the wavenumber, for the same simulations as in Figure 4. The colors and contours indicate the normalized error energy level.

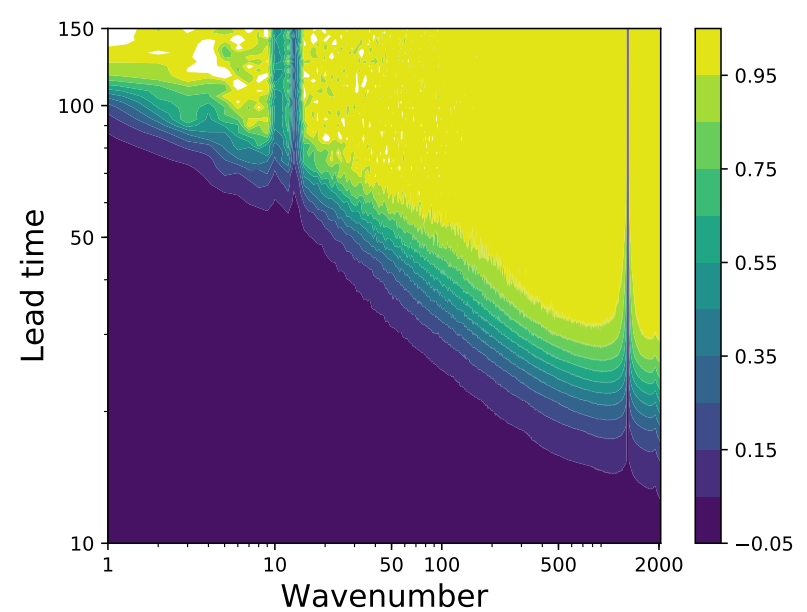

(b) 


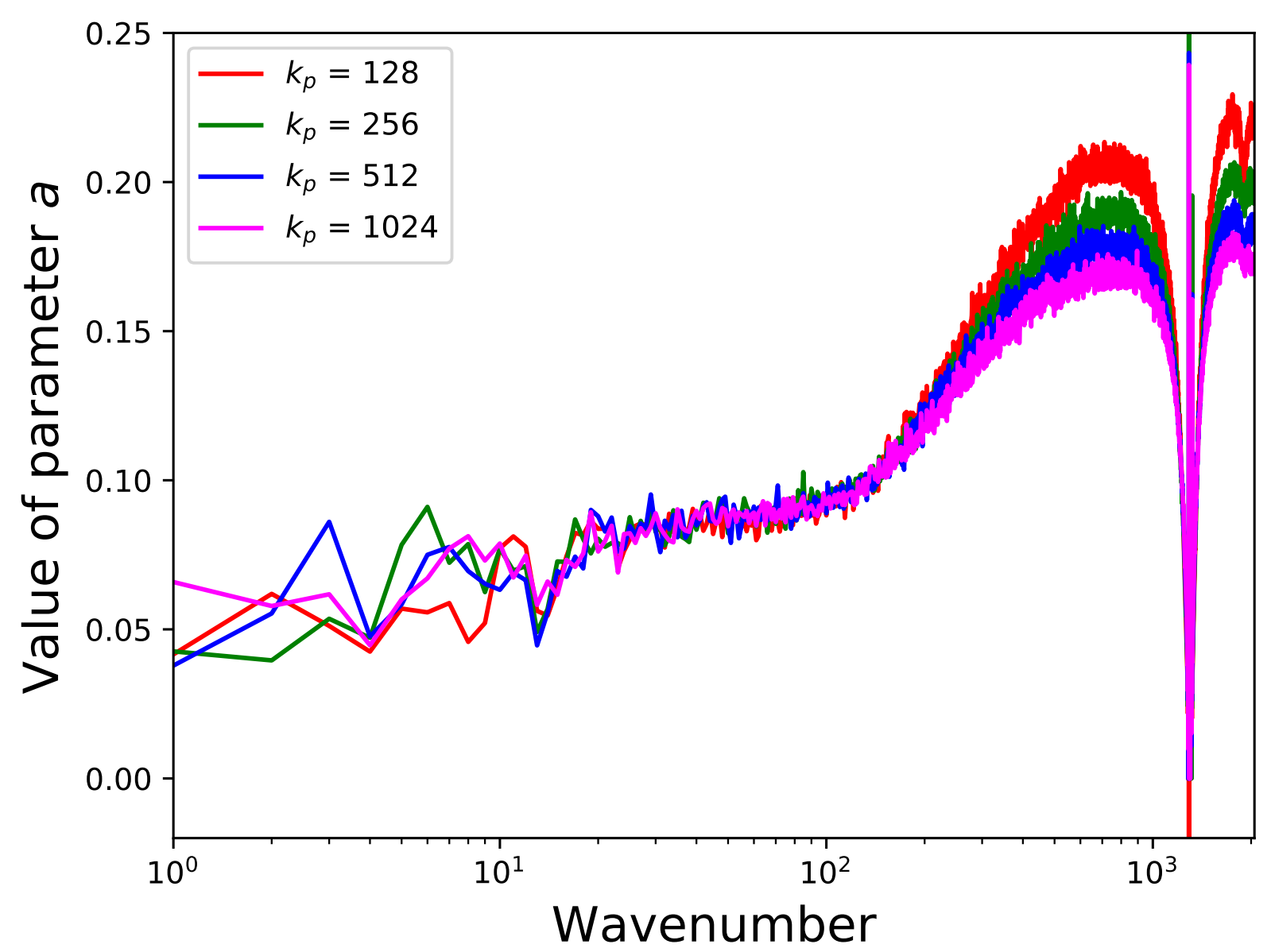

FIG. 6. Parameter $a$ of the Žagar model, fitted to the normalized error energy at individual wavenumbers according to equation (3), as a function of the wavenumber, for perturbation experiments of various $k_{p}$ for the highest-resolution model $k_{t}=2048$. The data are averaged over 5 independent realizations before the fitting is performed. Note that the vertical axis is linear and not logarithmic. 


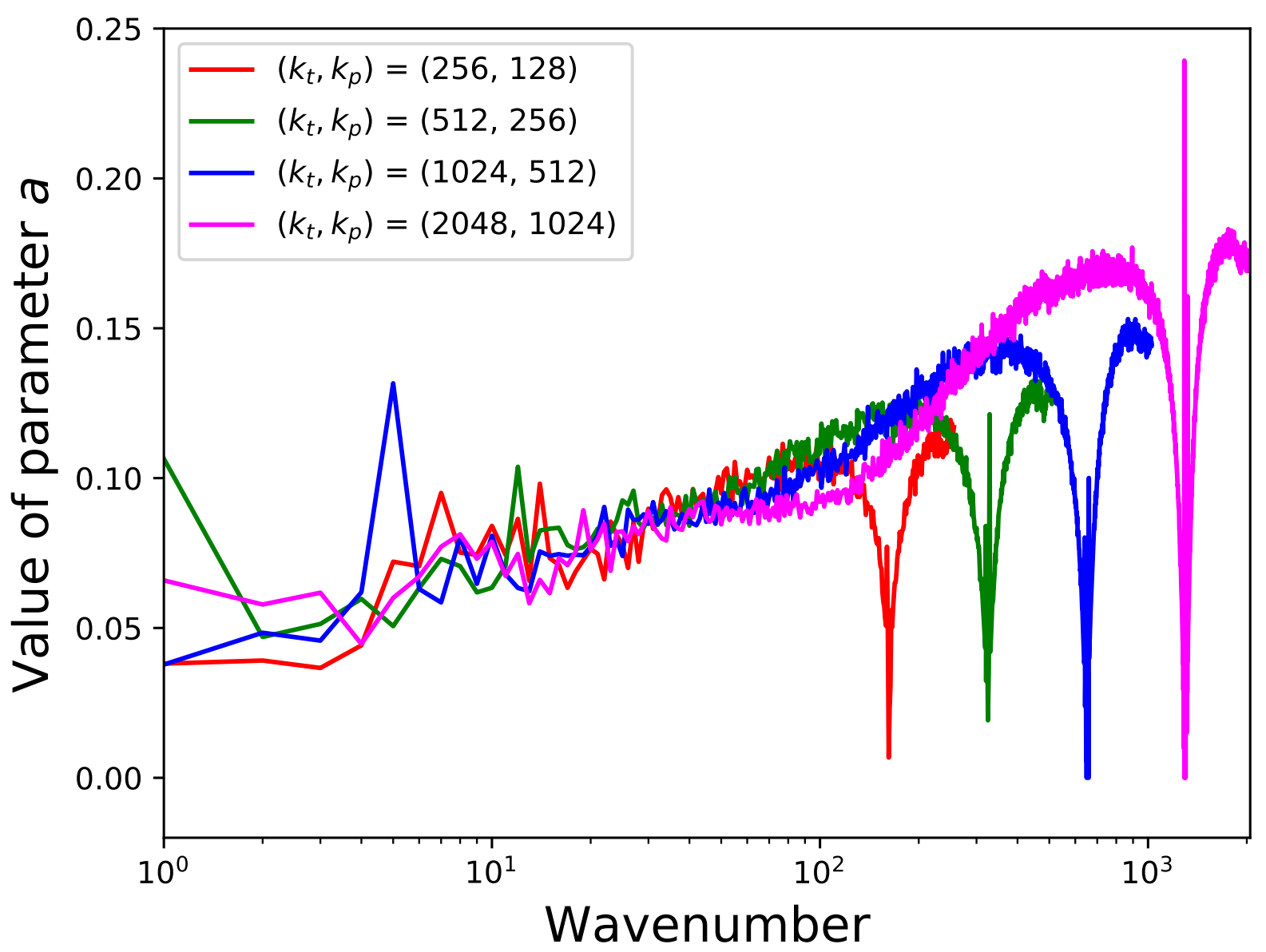

FIG. 7. As in Figure 6, but for combinations of $\left(k_{t}, k_{p}\right)$ such that $k_{p}=\frac{1}{2} k_{t}$. 


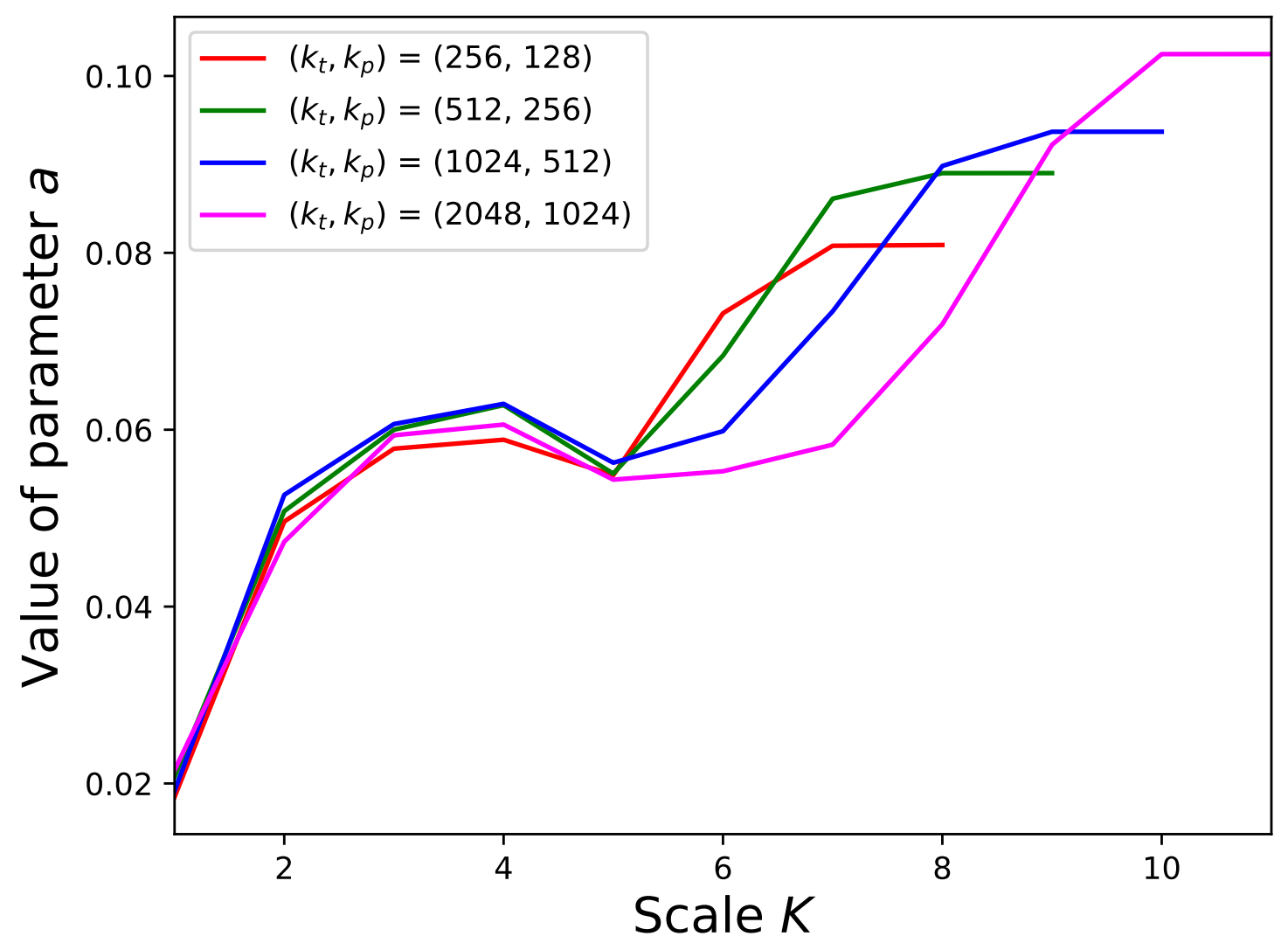

FIG. 8. As in Figure 7, but for the Lorenz (1969) model. 


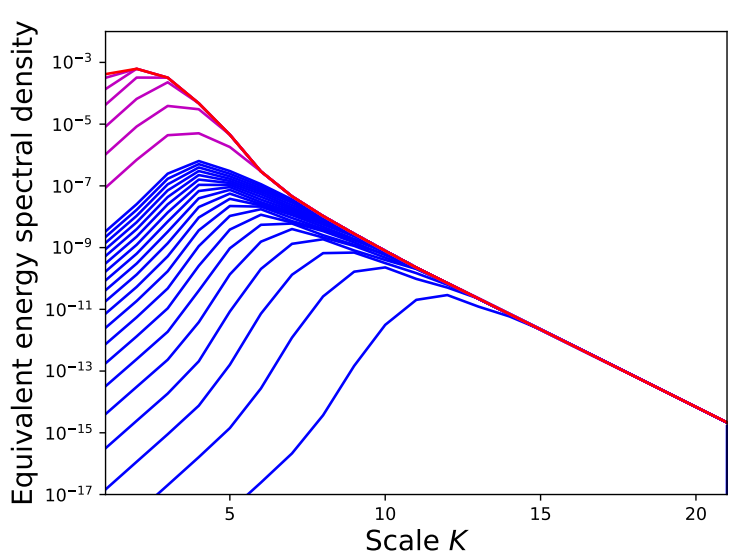

(a)

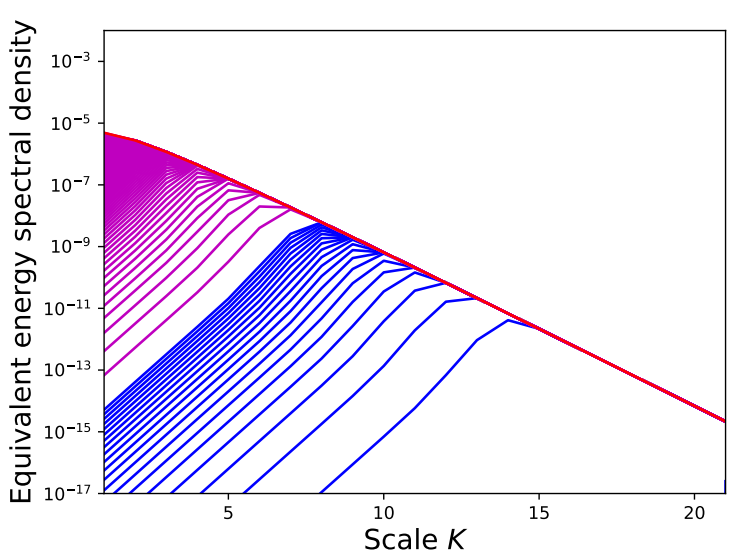

(b)

FIG. 9. (a) Evolution of the error energy spectrum (blue and magenta, from bottom to top) in the Lorenz (1969) model under the control energy spectrum (red) recovered from the $\left(k_{t}, k_{p}\right)=(2048,1024)$ simulations in Section 2 (with modifications, details of which are given in the text) and extended to $K_{\max }=21$ via equation (8), and an initial condition of $Z\left(K_{\max }\right)=5 \times 10^{-7} \times \sum_{L=1}^{K_{\max }} X(L)$ and $Z(K)=0$ for all other $K$. (b) As in (a), but for a single-range $k^{-\frac{5}{3}}$ control energy spectrum according to equation (9) yet normalized to such a level that the magnitude of the mesoscale part of the spectrum coincides with (a). The error spectra are plotted in blue at equal time-intervals of $\Delta t=3$ up to $t=60$, and in magenta at intervals of $\Delta t=30$ thereafter. The vertical axes show the equivalent energy spectral density $2^{-K} Z(K)$, a function that smoothly distributes $Z(K)$ which would have been a density in $k$ had $K$ been a continuous variable. 


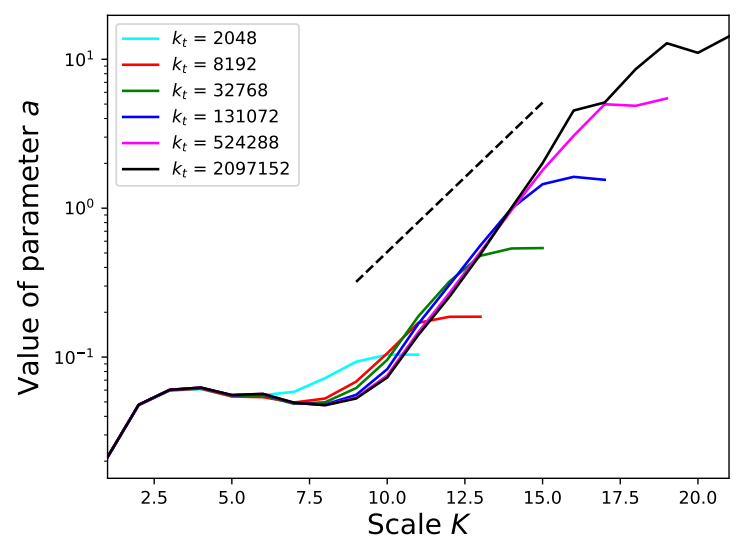

(a)

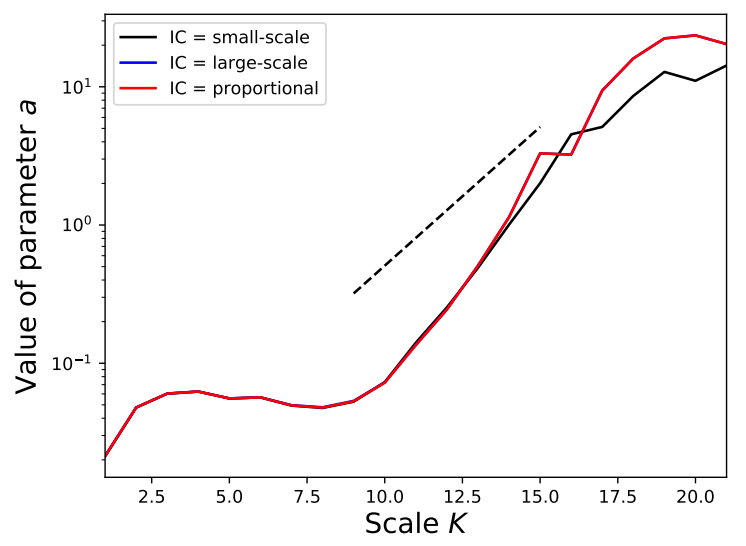

(b)

FIG. 10. (a) As in Figure 8, but for $K_{\max }=11$ (cyan), 13 (red), 15 (green), 17 (blue), 19 (magenta) and 21 (black), and an initial condition of $Z\left(K_{\max }\right)=5 \times 10^{-7} \times \sum_{L=1}^{K_{\max }} X(L)$ and $Z(K)=0$ for all other $K$. (b) shows the same black curve for the $K_{\max }=21$ simulation as (a), and additionally for cases where the initial condition of the same magnitude is moved to $K=1$ (red) or redistributed as a uniform fraction of the background spectrum (blue, which is essentially indistinguishable from the red). The vertical axes are logarithmic and the dashed lines indicate an appropriately normalized $2^{\frac{2}{3} K}$ scaling. 


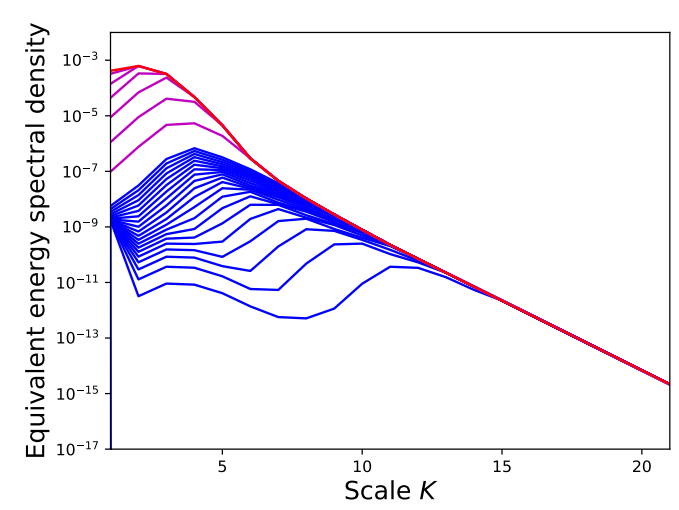

(a)

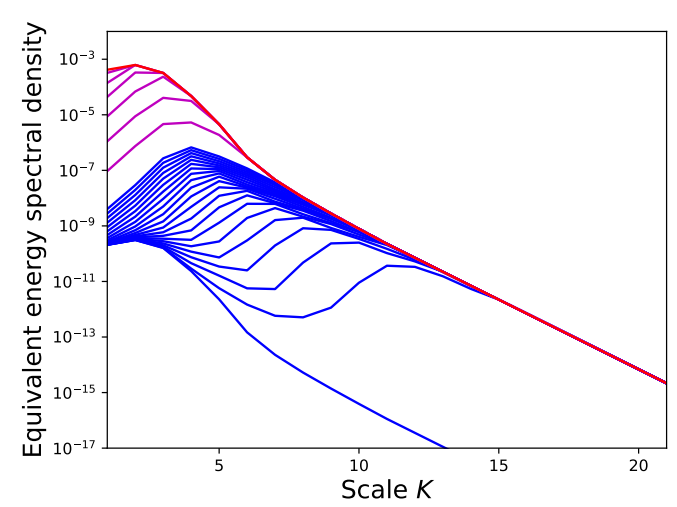

(b)

FIG. 11. As in Figure 9(a), but for the following initial conditions for $Z$ : (a) $Z(1)=5 \times 10^{-7} \times \sum_{L=1}^{K_{\max }} X(L)$ 566 and $Z(K)=0$ for all other $K$; (b) $Z(K)=5 \times 10^{-7} \times X(K)$ for all $K$. 